\title{
An improved molecular tool for screening bacterial colonies using GFP expression enhanced by a Dictyostelium sequence
}

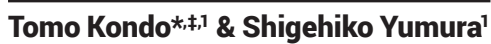

\section{ABSTRACT}

During molecular cloning, screening bacterial transformants is a timeconsuming and labor-intensive process; however, tractable tools that can be applied to various vectors for visual confirmation of desired colonies are limited. Recently, we reported that translational enhancement by a Dictyostelium gene sequence (TED) boosted protein expression even without an expression inducer in Escherichia coli. Here, we demonstrate a generally applicable molecular tool using the expression of green fluorescent protein enhanced by TED. By inserting a module related to TED into the cloning site in advance, we effectively screened $E$. coli colonies harboring the desired plasmid functions in a prokaryote (Magnetospirillum gryphiswaldense) or eukaryote (Dictyostelium discoideum). Thus, our system represents a user-friendly technique for cloning.

\section{METHOD SUMMARY}

This work presents a simple, reliable and cost-effective bacterial colony screening method using green fluorescent protein (GFP) expression. No expression inducer is required for GFP expression. In addition, this method is easily applied to any vector by insertion of a module related to GFP expression.

\section{KEYWORDS}

Dictyostelium • E. coli • GFP • Magnetospirillum $\cdot$ molecular cloning $\cdot$ screening

'Graduate School of Sciences \& Technology for Innovation, Yamaguchi University, 753-8512 Yamaguchi, Japan; *Author for correspondence: tomokondo@bio.c.u-tokyo.ac.jpl; ¥Present address: Department of Life Sciences, Graduate School of Arts \& Sciences, The University of Tokyo, Tokyo 153-8902, Japan

BioTechniques 68: 97-95 (February 2020) 10.2144/ btn-2019-0127
Molecular cloning is a technique used to create DNA constructs for studies in various biological fields, including molecular cell biology, biotechnology and synthetic biology. Because of extensive studies using Escherichia coli, many versatile methods are available for molecular cloning [1]; however, screening methods for bacterial transformants that contain the desired plasmid are limited.

A popular color-based screening method is blue-white screening using the pUC vector series [2,3]. The vector contains the $\alpha$-peptide gene [4] in the multi-cloning site (MCS). After its translation, followed by complementation of the $\beta$-galactosidase, blue pigments are produced through hydrolysis of the colorless lactose analog 5-bromo-4chloro-3-indolyl- $\beta$-d-galactoside (X-gal). When the $\alpha$-peptide gene is disrupted by insertion of a DNA fragment into the MCS, the colony appears white. Because $\alpha$-peptide expression is controlled by the lac operon, it can be upregulated by the addition of isopropyl $\beta$-d-thiogalactopyranoside (IPTG) [5]. Although this method is simple, various factors cause false-positive results, including the presence of an intact $\alpha$-peptide gene without corresponding product synthesis [1]. These false-positive results often result in unnecessary costs and labor because superfluous colonies are handled in the next procedure.

Alternatively, fluorescence-based screening has been developed for colony screening [6-11] and is based on the functionality of the green fluorescent protein (GFP), even in E. coli[12]. A nonfluorescent colored protein (i.e., chromoprotein) has also been used [13]. Using these visual markers, the screening enables efficient selection of colonies containing the desired vector inserts; however, these protein expressions often require an expression inducer such as IPTG, and the general feasibility of this method for a variety of vectors has not yet been reported.

Here, we present a tractable tool for bacterial colony screening by GFP fluorescence based on translational enhancement by a Dictyostelium gene sequence (TED) that enhances protein expression using a gene sequence of the social amoeba Dictyostelium discoideum in E. coli [14]. In our previous work, insertion of a Dictyostelium gene sequence (e.g., $m / c R$ ) upstream of the GFP gene can spontaneously induce visible levels of GFP expression without the addition of IPTG. We applied this method to generate shuttle vectors for the expression of heterologous proteins in magnetotactic bacteria or $D$. discoideum. We propose to incorporate this tool into a variety of vectors to raise the efficiency of colony screening.

\section{MATERIALS \& METHODS}

\section{E. coli culture \& transformation}

E. coli K12 strain HST08 Premium (Takara Bio, Shiga, Japan) was used for cloning. Lysogeny broth (LB) medium was obtained from Nippon Genetics (Tokyo, Japan) and supplemented with $100 \mu \mathrm{g} / \mathrm{ml}$ ampicillin. After chemical transformation [15,16], cells were selectively grown on LB agar prepared by solidifying the LB media with $1.5 \%$ agar supplemented with $100 \mu \mathrm{g} / \mathrm{ml}$ ampicillin.

\section{Magnetotactic bacteria culture \& transformation}

Magnetospirillum gryphiswaldense MSR-1 was obtained from DSMZ (Braunschweig, Germany). Cells were cultured at $28^{\circ} \mathrm{C}$ in DS medium containing $2.38 \mathrm{~g}$ HEPES (Dojindo Laboratories, Kumamoto, Japan), 3 g sodium pyruvate (Wako Pure Chemical Industries, Osaka, Japan), $0.1 \mathrm{~g}$ yeast extract (Oxoid, Basingstoke, UK), $3 \mathrm{~g}$ soybean peptone (Sigma-Aldrich, MO, 
(A)

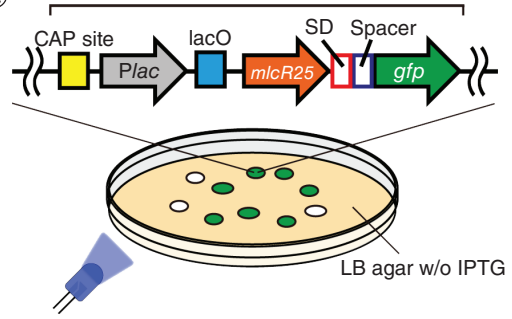

(C)

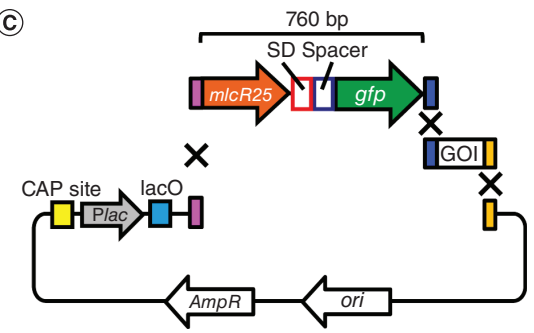

(B)

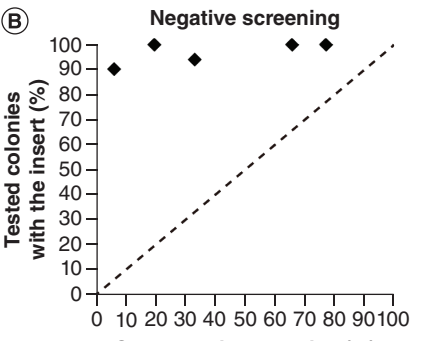

GFP-negative colonies (\%)

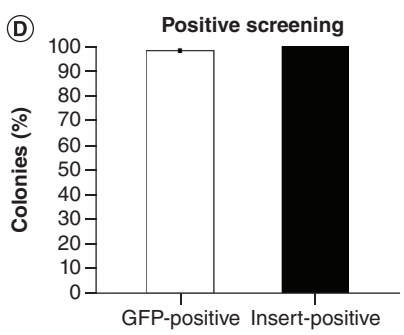

Figure 1. GFP fluorescence-based colony screening. (A) A schematic representation showing colonies with or without GFP expression under blue light-emitting diode illumination. GFP-expressing colonies carry plasmids containing the TED module, which contains the CAP binding site, lac promoter (Plac), lac operator (lacO), 25-bp fragment of the $3^{\prime}$ end of $m / c R$ ( $m / c R 25$ ), SD sequence, spacer and $g f p$. (B) Success rate of screening colonies with the insert (tubA) among GFP-negative colonies. A ligation reaction was carried out with (black rhombuses) or without an insert and used for transformation. The dashed line shows the theoretical success rate when the TED module is not used. In these experiments, fewer than $3 \%$ of colonies were observed as false positive (GFP-negative without the target insertion) $(n \geq 8)$. (C) A schematic representation depicting the plasmid design for GFP-positive colony selection. $m / c R 25$ indicates the 25-bp fragment of the $3^{\prime}$ end of $m / c R$. (D) The percentages of GFP-positive colonies (white) and colonies with successful insertion of the target (mRuby3 gene) among GFP-positive colonies (black). CAP: Catabolite activator protein; LB: Lysogeny broth; GFP: Green fluorescent protein; GOI: Gene of interest; SD: Shine-Dalgarno; TED: Translational enhancement by a Dictyostelium gene sequence.

USA), $0.34 \mathrm{~g} \mathrm{NaNO}_{3}$ (Wako Pure Chemical Industries), $0.1 \mathrm{~g} \mathrm{KH}_{2} \mathrm{PO}_{4}$ (Wako Pure Chemical Industries), $0.15 \mathrm{~g} \mathrm{MgSO}_{4} \cdot 7 \mathrm{H}_{2} \mathrm{O}$ (Wako Pure Chemical Industries), and $3 \mathrm{ml}$ of 10-mM ferric quinate in $1 \mathrm{I}$ of distilled water, a procedure that was slightly modified from a previous study [17]. The $\mathrm{pH}$ of DS medium was adjusted to 7.0 with $\mathrm{NaOH}$. Ferric quinate (10-mM) was prepared by adding $4.5 \mathrm{~g}$ of $\mathrm{FeCl}_{3} \cdot 6 \mathrm{H}_{2} \mathrm{O}$ (Kanto Chemical, Tokyo, Japan) and $1.9 \mathrm{~g}$ of quinic acid (Sigma-Aldrich) to $1 \mathrm{I}$ of distilled water. For agar-plate culture, $1 \mathrm{~g} / \mathrm{l}$ activated charcoal (Wako Pure Chemical Industries) was added to the above DS medium. After autoclaving, 1,4-dithiothreitol (final concentration of $1 \mathrm{mM}$ ) was added aseptically and solidified by adding $1.5 \%$ agar. Antibiotics ( $5 \mu \mathrm{g} / \mathrm{ml}$ ampicillin) were supplemented, as needed.

Transformation was carried out by electroporation. For each pulsing experiment, $15 \mathrm{ml}$ of exponential phase cells grown in $15-\mathrm{ml}$ polypropylene tubes were collected by centrifugation $(1200 \times g$ for $10 \mathrm{~min}$ ) at $4^{\circ} \mathrm{C}$. The cells were washed twice using cold electroporation buffer (1-mM HEPES, pH 7.0; 1-mM MgCl ; 272-mM sucrose) and resuspended in about $50 \mu$ of the same buffer. The mixture was loaded into a chilled $0.1-\mathrm{cm}$ cuvette with plasmid DNA and subjected to a single pulse using the Gene Pulser Xcell ${ }^{\text {TM }}$ system (Bio-Rad Laboratories, CA, USA) set at $25 \mu \mathrm{F}$ capacitance, $200 \Omega$ resistance and $0.8-1.0 \mathrm{kV}$ voltage. The cells were recovered in $1 \mathrm{ml}$ of fresh DS medium overnight at $28^{\circ} \mathrm{C}$ and then plated onto a DS medium agar plate with antibiotics. For approximately 1 week, visible colonies were observed. After checking the colonies by PCR using EmeraldAmp ${ }^{\circledR}$ MAX (Takara Bio), the selected cells were grown in DS medium with antibiotics. Plasmids were purified using NucleoSpin ${ }^{\circledR}$ Plasmid (MachereyNagel, Düren, Germany) following the manufacturer's instructions.
Dictyostelium culture \& transformation $D$. discoideum cells of the $A \times 2$ were grown in HL5 medium ( $15.4 \mathrm{~g}$ glucose, $7.15 \mathrm{~g}$ yeast extract, $14.3 \mathrm{~g}$ bacteriological peptone, $0.486 \mathrm{~g} \mathrm{KH}_{2} \mathrm{PO}_{4}$ and $1.28 \mathrm{~g} \mathrm{Na}_{2} \mathrm{HPO}_{4} \cdot 12 \mathrm{H}_{2} \mathrm{O}$ per liter) at $22^{\circ} \mathrm{C}$ [18]. Additional antibiotics (G418, blasticidin S, streptomycin and ampicillin) were supplemented at appropriate concentrations, as needed. Transformation was carried out by electroporation as described previously [19] with modifications. Briefly, cells were washed with electroporation buffer (50-mM sucrose, $10-\mathrm{mM}$ $\mathrm{Na}-\mathrm{K}$-phosphate buffer, $\mathrm{pH}$ 6.1) and collected by centrifugation ( $536 \times g$ for $2 \mathrm{~min}$ ) at $4^{\circ} \mathrm{C}$. After removing the supernatant, cells were suspended in about $100 \mu \mathrm{l}$ of the same buffer. The mixture was loaded into a chilled $0.1-\mathrm{cm}$ cuvette with plasmid DNA and subjected to two square pulses at 5-s intervals using the Gene Pulser Xcell system set at $0.55-\mathrm{kV}$ voltage. The cells were recovered in $\mathrm{HL} 5$ overnight at $22^{\circ} \mathrm{C}$, and the appropriate antibiotics were added for selection.

\section{Plasmids}

Plasmids used in the study were constructed using standard techniques. For seamless cloning, In-Fusion ${ }^{\circledast}$ cloning (Takara Bio) was used following the manufacturer's protocols. The pMGT fragment [20], msp3 promoter [21], mms13 [22] and HaloTag gene [23] were synthesized by Genewiz (NJ, USA). The gene that encodes the HaloTag protein was optimized for Magnetospirillum codons with permission from Promega Corporation (WI, USA). The neo gene encodes aminoglycoside 3'-phosphotransferase with the promoter, and the terminator and fragment of Ddp1 [24] were cloned from the pBIG vector (GenBank accession number AF270470) [25]. The actin15 promoter and terminator with MCS were synthesized by Genewiz. The genes that encode blasticidin-S deaminase, mEGFP (referred to as simply GFP), and mRuby3 were synthesized with codon optimization for Dictyostelium (FASMAC, Kanagawa, Japan; Genewiz).

\section{Imaging}

For excitation of GFP on an agar plate, a custom-made blue light-emitting diode illuminator was used. For imaging of magnetotactic bacteria, cells were 
collected by centrifugation $(4300 \times g$ for $3 \mathrm{~min})$ at $25^{\circ} \mathrm{C}$ and then washed twice with distilled water. The cells were incubated with Promega ${ }^{\mathrm{TM}}$ HaloTag $^{\circledR}$ TMR Ligand $(1-\mu \mathrm{M}$ at the final concentration) in distilled water at $25^{\circ} \mathrm{C}$ for $1 \mathrm{~h}$ in the dark. After being washed twice, cells were added onto a coverslip $(24 \times 60 \mathrm{~mm})$ and overlaid with a block of $1.5 \%$ agar $(8 \times 8 \times 1 \mathrm{~mm})$ in distilled water. Fixed Dictyostelium cells were prepared as described previously [26]. The specimens were observed with a microscope (Diaphot 300 or Eclipse Ti; Nikon, Tokyo, Japan) equipped with either an Orca-ER C4742-80-12AG (Hamamatsu Photonics, Hamamatsu, Japan) or a Zyla 4.2 (Andor Technology, Belfast, Northern Ireland) camera and a mercury lamp (Nikon) using a $100 \times$ objective lens. Fluorescence in magnetotactic bacteria was evaluated using a custom-written macro in Fiji [27].

\section{RESULTS \& DISCUSSION}

\section{Colony screening using TED-induced}

\section{GFP expression}

We developed a GFP expression vector in combination with TED without any adverse effects such as growth rate [14]. In this system, $g f p$, which is placed downstream of the 25-bp fragment of the $3^{\prime}$ end of $m / c R$ ( $m / c R 25$ ) and the Shine-Dalgarno sequence, is expressed under control of the lac promoter, but IPTG is not necessary. Even in the absence of IPTG, fluorescence can be readily distinguished by excitation with a blue light-emitting diode. The sequence region necessary for GFP expression is hereinafter referred to as the TED module (Figure 1A).

First, we tested a negative screening method to detect successful target insertion by the loss of GFP fluorescence. Part of the TED module (m/cR25-SD sequence-spacer-gfp) in the pUC19 vector was replaced with an $\mathrm{Xbal}-\mathrm{BamHI}$ fragment of the target sequence encoding D. discoideum $\alpha$-tubulin (1395 bp) as a representative. We obtained plates with various ratios of GFP-negative (i.e., target-inserted) and GFP-positive (i.e., empty-vector) colonies by varying the insert concentration used for DNA ligation (Figure 1B, $x$-axis). We found that more than $90 \%$ of GFP-negative colonies were successfully inserted in any condition (Figure 1B,y-axis).

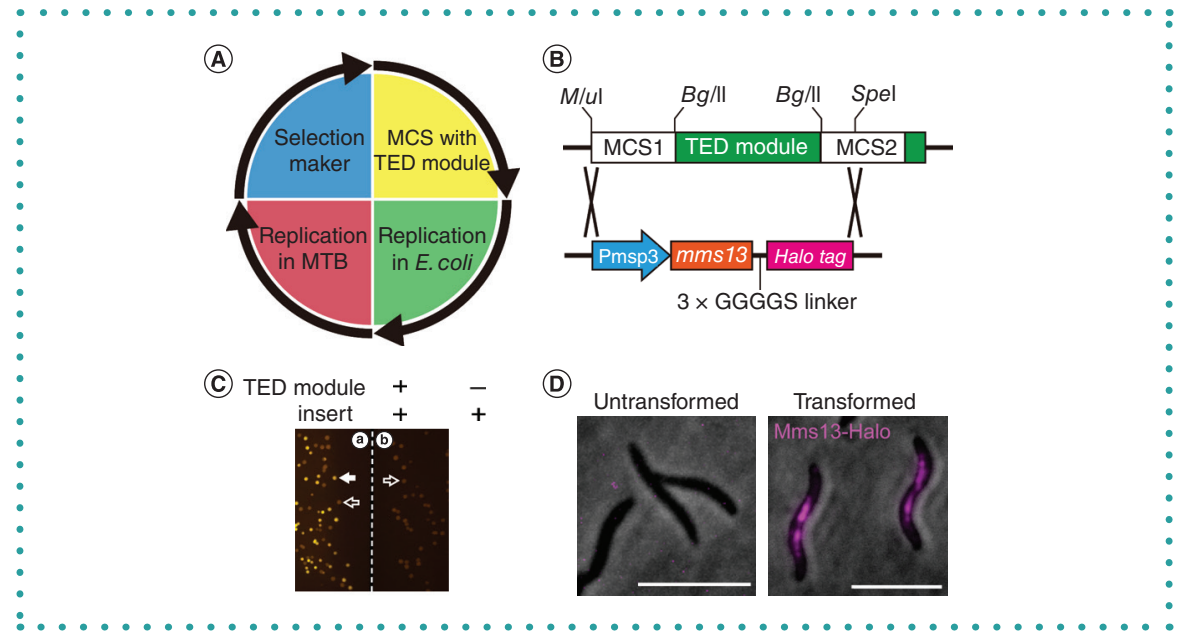

Figure 2. Negative screening of a shuttle vector for magnetotactic bacteria and visualization of their magnetosomes. (A) Schematic overview of the shuttle vector with four modules shown in different colors: yellow $=$ MCS with the TED module; green = Escherichia coli replication module; red = MTB replication module; blue = selection marker. (B) The sequence containing the $m s p 3$ promoter (Pmsp3), mms13 and the HaloTag gene was inserted into the Mlul-Spel site. (C) Representative image of $E$. coli colonies cultured on an agar plate in the presence (a) or absence (b) of the TED module. Representative colonies with (white arrow) or without (open arrows) GFP expression are marked. (D) Image of MTB MSR-1 stained with the HaloTag TMR Ligand. Bars: $5 \mu \mathrm{m}$. GFP: Green fluorescent protein; MCS: Multi-cloning site; MTB: Magnetotactic bacteria; TED: Translational enhancement by a Dictyostelium gene sequence.

For negative controls, without addition of the insert with the vector in the ligation mixture, GFP-negative colonies were observed at a rate of $\sim 3 \%$. Under these conditions, even for a 'worst' plate with $6 \%$ of colonies showing no GFP fluorescence, we could select colonies containing the target sequence with $90 \%$ probability. Thus, these results suggest that this method has sufficient sensitivity and high reliability.

We also tested a positive screening method to detect successful target insertions by the appearance of GFP fluorescence. Seamless cloning is a powerful method to generate constructs; however, there are few tools for visually confirming desired colonies on the plate, except for antibiotic selection. Thus, we seamlessly cloned the TED module into the pUC19 vector with the sequence of a gene of interest (GOI) (Figure 1C). In this case, $98 \pm 0.6 \%$ of colonies ( $n=7031$ from six independent experiments) showed the expected GFP fluorescence at a level that could be determined visually. Among them, we could select colonies with the inserted target with $100 \%$ probability $(n=96)$ (Figure 1D). Therefore, this TED-based expression system is an effective tool for both negative and positive colony screening.
A TED-module-combined shuttle vector for magnetotactic bacteria

We applied the TED-based screening system to create a shuttle vector between $E$. coli and another prokaryote. A previous study reported a vector combined with $\mathrm{pUC} 19$ and a cryptic plasmid isolated from the magnetotactic bacterium Magnetospirillum magneticum [20]. Based on this, we designed a vector that contains the fragment necessary for its replication in magnetotactic bacteria and $E$. coli, a selection marker, and a fragment of the TED module inserted into the MCS (Figure 2A \& Supplementary Figure S1).

The TED module was replaced with nucleotides encoding the magnetosome protein Mms13-fused HaloTag under control of the $m s p 3$ promoter (Pmsp3) [22] to visualize magnetosomes (Figure 2B). The $E$. coli colonies carrying plasmids with the desired substitution were selected by GFP-negative screening (Figure 2C). Subsequently, the plasmid was transferred into a similar Magnetospirillum strain, MSR-1, and the transformed cells were observed. The fluorescence of HaloTag ligands in MSR-1 cells is shown as aligned dot signals along the long axis of the cell at the midline (Figure 2D), where the magnetosome chain is typically localized $[28,29]$. The $m m s 13$. 
(A)

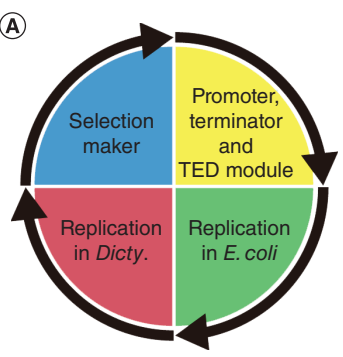

(c)

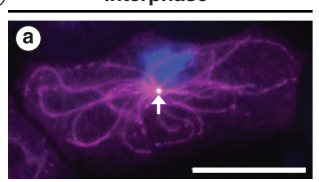

(B)

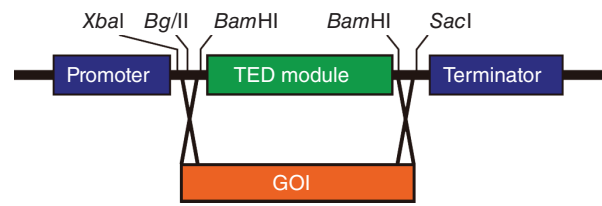

Mitotic phase

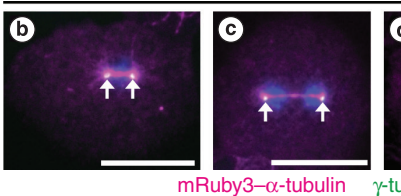

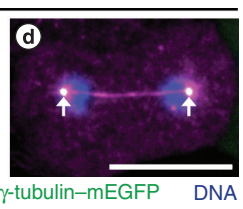

Figure 3. Negative screening of a shuttle vector for Dictyostelium and visualization of the cytoskeletal proteins. (A) Schematic overview of the extrachromosomal vector with the four modules shown in different colors: yellow = actin15 promoter and terminator spacing by the MCS with the TED module; green = Escherichia coli replication module; red = Dictyostelium replication module (a 2033-bp HindIII fragment of Ddp1); blue = selection marker for E. coli and Dictyostelium. (B) The sequence encoding the $\mathrm{GOI}$ was inserted into the $\mathrm{Xbal}-\mathrm{BamHI}$ site between the promoter and terminator. (C) Fluorescence images of fixed cells co-expressing mRuby $3-\alpha$-tubulin and $\gamma$-tubulin-GFP; arrows indicate the centrosome; (a) interphase, (b) metaphase, (c) anaphase and (d) telophase. Bars, $10 \mu \mathrm{m}$.

GFP: Green fluorescent protein; GOI: Gene of interest; MCS: Multi-cloning site; TED: Translational enhancement by a Dictyostelium gene sequence.

gene used here is derived from the AMB-1 strain and is known as mamC in the MSR-1 strain (80\% amino acid sequence identity). Our observations revealed that $m m s 13$ is still normally targeted to the magnetosomes, even in the MSR-1 strain.

To further test the functionality and stability of our plasmid, we cultured cells in the presence or absence of antibiotics. The signals of HaloTag ligands that bind to HaloTag in cells were detected by image processing (Supplementary Figure S2A). We found that our plasmid could function (Supplementary Figure S2B) and be isolated (Supplementary Figure S2C), even from cells cultured in an environment without selective pressure for more than 3 weeks. Considering that the doubling time of bacteria cultured in media is approximately $8 \mathrm{~h}$, the plasmids were stably maintained in the strain. Therefore, we concluded that our shuttle vector functions properly for protein expression in magnetotactic bacteria.

\section{A TED-module-combined shuttle vector for $D$. discoideum}

Next, we created a shuttle vector for eukaryotic $D$. discoideum in combination with the TED module. This vector consists of four components that are required for replication and selection in Dictyostelium or E. coli and expression of the target protein (Figure $3 \mathrm{~A}$ \& Supplementary Figure S3). The TED module was inserted into the MCS placed between the Dictyostelium actin15 promoter and terminator to prepare a system capable of GFP-negative screening (Figure 3B).

We created shuttle vectors to express well-known proteins. Alpha-tubulin and $\gamma$-tubulin are extensively characterized proteins that constitute microtubules and centrosomes, respectively, in various organisms, including Dictyostelium [30-35]. Two vectors were prepared by replacing the TED module with these target genes and genes encoding fluorescent proteins connecting a flexible (Gly-Gly-Gly-Gly-Ser) linker sequence [36]. After bacterial colony screening and transformation into Dictyostelium cells, localization of the expressed protein was observed. As expected, mRuby3- $\alpha$-tubulin showed a network structure, and $\gamma$-tubulin-GFP localized at the center of the network during interphase and mitosis, suggesting that the created vectors were functional (Figure $3 \mathrm{C}$ ).

Our vector was designed for simultaneous expression of multiple proteins from a single plasmid, which can reduce the time to generate transformants and suppress variations in their expression levels [37]. We introduced more than one desired GOI into the vector using In-Fusion cloning, which has high precision for cloning (Supplementary Figure S1). A generated vector contained multiple GOls arranged in tandem, separated by a promoter or terminator (Supplementary Figure S4A). The gene encoding mRuby3histone $\mathrm{H} 1$ (GOI2) [38,39] was additionally incorporated into the plasmid containing the first gene encoding $\gamma$-tubulin-GFP (GOI1) with the promoter and terminator. Similar to a cell with the single expression vector, the centrosome was similarly visualized (Supplementary Figure S4B \& C). In addition, the nucleus was successfully visualized. Thus, our vector can be used for multiple protein expression after only one transformation. Taken together, our vectors can be used for heterologous protein expression in Dictyostelium.

\section{FUTURE PERSPECTIVE}

Here, we present a GFP-based method for screening colonies with the desired insert by inserting the TED module into various vectors. This new green-white screening method will aid in the routine construction of many vector types used in biological science studies. Thus, using TED with a wide variety of vectors, it is possible to provide a user-friendly process, construct many vectors with less effort, and finally increase the efficiency of experiments.

\section{SUPPLEMENTARY DATA}

To view the supplementary data that accompany this paper please visit the journal website at: www.future-science. com/doi/suppl/10.2144/btn-2019-0127

\section{AUTHOR CONTRIBUTIONS}

$\mathrm{T}$ Kondo performed the experiments. $\mathrm{T}$ Kondo and $S$ Yumura contributed to the writing of the manuscript. All of the authors contributed to the conception and design of the study and analysis and interpretation of the data.

\section{ACKNOWLEDGMENTS}

We thank Drs. Yoshihiro Fukumori and Azuma Taoka (Kanazawa University, Japan) for providing protocols for handling magnetotactic bacteria and Dr. Yuki Hara (Yamaguchi 
University, Japan) for the use of his microscope. Codon optimization of the nucleotide sequence that encodes the HaloTag protein was performed with permission from Promega Corporation.

\section{FINANCIAL \& COMPETING INTERESTS DISCLOSURE}

T Kondo was supported by the Japan Society for the Promotion of Science Research Fellowships for Young Scientists. This research was supported by the Japan Society for the Promotion of Science (KAKENHI grant nos. 16J08310 and $19 K 15809$ to $T$ Kondo). The authors have no other relevant affiliations or financial involvement with any organization or entity with a financial interest in or financial conflict with the subject matter or materials discussed in the manuscript apart from those disclosed.

No writing assistance was utilized in the production of this manuscript.

\section{ETHICAL CONDUCT OF RESEARCH}

This article does not contain any studies with human participants or animals performed by any of the authors.

\section{OPEN ACCESS}

This work is licensed under the AttributionNonCommercial-NoDerivatives 4.0 Unported License. To view a copy of this license, visit http://creativecommons.org/licenses/ by-nc-nd/4.0/

\section{REFERENCES}

Papers of special note have been highlighted as:

- of interest; $\cdot$ - of considerable interest

1. Green MR, Sambrook J. Molecular Cloning: A Laboratory Manual (4th Edition). Cold Spring Harbor Laboratory Press, NY, USA (2012)

2. Messing J, Gronenborn B, Müller-Hill B, Hopschneider $\mathrm{PH}$. Filamentous coliphage $\mathrm{M} 13$ as a cloning vehicle: insertion of a Hindll fragment of the lac regulatory region in M13 replicative form in vitro. Proc. Natl Acad. region in M13 replicative form in vitro.
Sci. USA 74(9), 3642-3646 (1977).

3. Yanisch-Perron C, Vieira J, Messing J. Improved M13 phage cloning vectors and host strains: nucleotide sequences of the M13mpl8 and pUC19 vectors. Gene 33(1), 103-119 (1985).

4. Ullmann A, Jacob F, Monod J. Characterization by in vitro complementation of a peptide corresponding to an operator-proximal segment of the $\beta$-galactosidase structural gene of Escherichia coli. J. Mol. Biol. 24(2), 339-343 (1967).

5. Jacob F, Monod J. Genetic regulatory mechanisms in the synthesis of proteins. J. Mol. Biol. 3, 318-356 (1961).

6. Inouye $\mathrm{S}$, Ogawa $\mathrm{H}$, Yasuda $\mathrm{K}$, Umesono K, Tsuji FI. A bacterial cloning vector using a mutated Aequorea green fluorescent protein as an indicator. Gene 189(2), 159-162 (1997).

- Shows that GFP expression in Escherichia coli can be used for desired colony selection.

7. Truong K, Khorchid A, Ikura M. A fluorescent cas- sette-based strategy for engineering multiple domain fusion proteins. BMC Biotechnol. 3, 1-8 (2003).

8. Tang J, Liang S, Zhang J, Gao Z, Zhang S. pGreen-S: a clone vector bearing absence of enhanced green fluorescent protein for screening recombinants. Anal. Biochem. 388(1), 173-174 (2009).

9. Banerjee S, Kumar J, Apte-Deshpande A, Padmanabhan $S$. A novel prokaryotic vector for identification and selection of recombinants: direct use of the vector for expression studies in E. coli. Microb. Cell. Fact. 9, 30 (2010).

10. Wong SSC, Truong K. Fluorescent protein-based methods for on-plate screening of gene insertion. PIOS ONE 5(12), e14274 (2010).

11. Speltz EB, Regan $L$. White and green screening with circular polymerase extension cloning for easy and reliable cloning. Protein Sci. 22(6), 859-864 (2013).

12. Chalfie M, Tu Y, Euskirchen G, Ward WW, Prasher DC. Green fluorescent protein as a marker for gene expression. Science 263(5148), 802-805 (1994).

13. Tafoya-Ramírez MD, Padilla-Vaca $F$, Ramírez-Saldaña AP et al. Replacing standard reporters from molecular cloning plasmids with chromoproteins for positive clone selection. Molecules 23(6), E1328 (2018).

14. Kondo T, Yumura S. Translation enhancement by a Dictyostelium gene sequence in Escherichia coli. Appl. Microbiol. Biotechnol. 103(8), 3501-3510 (2019).

.. Describes the expression enhancement activity of Dictyostelium gene sequences.

15. Chung CT, Niemela SL, Miller RH. One-step preparation of competent Escherichia coli: transformation and storage of bacterial cells in the same solution. Proc. Nat Acad. Sci. USA 86(7), 2172-2175 (1989).

16. Inoue $H$, Nojima $H$, Okayama $H$. High efficiency transformation of Escherichia coli with plasmids. Gene 96(1) 23-28 (1990).

17. Schultheiss D, Schüler D. Development of a genetic system for Magnetospirillum gryphiswaldense. Arch. Microbiol. 179(2), 89-94 (2003).

18. Watts DJ, Ashworth JM. Growth of myxameobae of the cellular slime mould Dictyostelium discoideum in axenic culture. Biochem. J. 119(2), 171-174 (1970).

19. Faix J, Kreppel L, Shaulsky G, Schleicher M, Kimmel AR. A rapid and efficient method to generate multiple gene disruptions in Dictyostelium discoideum using a single selectable marker and the Cre-loxP system. Nucleic Acids Res. 32(19), e143 (2004).

20. Okamura $Y$, Takeyama $H$, Sekine $T$ et al. Design and application of a new cryptic-plasmid-based shuttle vector for Magnetospirillum magneticum. Appl. Environ. Microbiol. 69(7), 4274-4277 (2003).

21. Yoshino T, Matsunaga T. Development of efficient expression system for protein display on bacterial magnetic particles. Biochem. Biophys. Res. Comm. 338(4), 1678-1681 (2005)

22. Yoshino T, Matsunaga T. Efficient and stable display of functional proteins on bacterial magnetic particles using $\mathrm{mms} 13$ as a novel anchor molecule. Appl. Environ. Microbiol. 72(1), 465-471 (2006).

23. Ohana RF, Encell LP, Zhao K et al. HaloTag7: a genetically engineered tag that enhances bacterial expression of soluble proteins and improves protein purification. Protein Expr. Purif. 68(1), 110-120 (2009).

24. Firtel RA, Silan C, Ward TE et al. Extrachromosomal replication of shuttle vectors in Dictyostelium discoideum. Mol. Cell. Biol. 5(11), 3241-3250 (1985).

25. Egelhoff TT, Lee RJ, Spudich JA. Dictyostelium myosin heavy chain phosphorylation sites regulate myosin filament assembly and localization in vivo. Cell 75(2), 363-371 (1993)

26. Yumura S, Mori H, Fukui Y. Localization of actin and myosin for the study of ameboid movement in Dictyostelium using improved immunofluorescence. J. Cell Biol. 99(3), 894-899 (1984).

27. Schindelin J, Arganda-Carreras I, Frise E et al. Fiji: an open-source platform for biological-image analysis. Nat Methods 9(7), 676-682 (2012)

28. Schleifer KH, Schüler D, Spring S et al. The genus Magnetospirillum gen. nov. description of Magnetospirillum gryphiswaldense sp. nov. and transfer of Aquaspirillum magnetotacticum to Magnetospirillum magnetotacticum comb. nov. Syst. Appl. Microbiol. 14(4), 379-385 (1991).

29. Lang C, Schüler D. Expression of green fluorescent protein fused to magnetosome proteins in microaerophilic magnetotactic bacteria. Appl. Environ. Microbiol. 74(15), 4944-4953 (2008)

30. White E, Tolbert EM, Katz ER. Identification of tubulin in Dictyostelium discoideum: characterization of some unique properties. J. Cell Biol. 97(4), 1011-1019 (1983).

31. Kitanishi T, Shibaoka H, Fukui Y. Disruption of microtubules and retardation of development of Dictyostelium with ethyl $\mathbf{N}$-phenylcarbamate and thiabendazole.
Protoplasma 120(3), 185-196 (1984).

32. Kitanishi-Yumura T, Blose SH, Fukui Y. Role of the MT-MTOC complex in determination of the cellular ocomotory unit in Dictyostelium. Protoplasma 127, 133-146 (1985).

33. Kitanishi-Yumura T, Fukui Y. Reorganization of microtubules during mitosis in Dictyostelium: dissociation from MTOC and selective assembly/disassembly in situ. Cell Motil. Cytoskeleton 8(2), 106-117 (1987).

34. Ueda M, Gräf R, MacWilliams HK, Schliwa M, Euteneuer U. Centrosome positioning and directionality of cell movements. Proc. Natl Acad. Sci. USA 94(18), 9674-9678 (1997)

35. Ueda M, Schliwa M, Euteneuer U. Unusual centrosome cycle in Dictyostelium: correlation of dynamic behavio and structural changes. Mol. Biol. Cell 10(1), 151-160 (1999)

36. Chen X, Zaro JL, Shen WC. Fusion protein linkers: property, design and functionality. Adv. Drug Deliv. Rev. 65(10), 1357-1369 (2013).

37. Veltman DM, Akar G, Bosgraaf L, van Haastert PJ. A new set of small, extrachromosomal expression vectors for Dictyostelium discoideum. Plasmid 61 (2), 110-118 (2009).

38. Hauser LJ, Dhar MS, Olins DE. Dictyostelium discoideum contains a single-copy gene encoding a unique subtype of histone H1. Gene 154(1), 119-122 (1995).

39. Nagasaki A, de Hostos EL, Uyeda TQ. Genetic and morphological evidence for two parallel pathways of cell-cycle-coupled cytokinesis in Dictyostelium. J. Cell Sci. 115(10), 2241-2251 (2002). 